\title{
Dermatology made easy, first edition
}

Dermatology Made Easy provides a concise, pocket-sized reference guide to any clinician with a focus on the practice of dermatology. The book is authored by associate professor Amanda Oakley, with whom some readers will be familiar from the hugely successful DermNetNZ.org resource. The author combines more than 3 decades of clinical experience with finesse in easy-to-digest information in this valuable resource. With a focus on high-yield dermatologic complaints, this book masterfully selects topics of importance and directs readers to the free online resource for further guidance or details on more complex topics.

The opening chapter introduces readers to a systematic diagnostic approach with a differential diagnostic tool categorized by symptoms, morphology, and body site, highlighting patterns of a disease and predilections for certain body sites to assist with diagnosis. This diagnostic approach will be new to some; however, it is well discussed in the text and will enhance many clinicians' mastery of dermatology concepts.

Each successive chapter provides a more in-depth analysis of common skin complaints, including infections, inflammatory rashes, noninflammatory conditions, and skin lesions, summarized and accompanied by high-quality clinical images. These conditions are further structured and explored by etiology, complications, management, and prognosis. The final chapter explores relevant investigations and treatments available for each condition covered, including pharmacologic and procedural approaches to management, providing a stepwise approach to the many treatment options available. Additionally, Oakley provides a useful template for conducting a dermatologic history and describes important visual clues to look for during an examination.

Oakley's text is not intended to cover rare and intriguing integumentary conditions; rather, it is targeted toward audiences needing a single resource for common complaints and important dermatologic emergencies. The resource is an excellent addition to any collection, particularly due to the high-quality summaries and clinical imagery. The book, however, would be most beneficial to juniors and clinicians with varying backgrounds interested in dermatology or those after a succinct pocket guide. The text is concise, well-structured, and supported by broader in-depth resources and commentary online. Dermatology Made Easy is an investment in your own career, enabling access to decades of high-quality experience within a pocket-sized resource.

\section{Nicholas Van Rooij, MD}

Brisbane, Southport, and St Lucia, Australia 